\title{
Channel Prediction Aided Multiuser Transmission in SDMA
}

\author{
W. Liu, L. L. Yang and L. Hanzo \\ School of ECS, University of Southampton, SO17 1BJ, United Kingdom. \\ Tel: +44-23-8059 6671, Fax: +44-23-8059 4508 \\ Email: $\{$ w103r,lly,lh\}@ecs.soton.ac.uk, http://www-mobile.ecs.soton.ac.uk
}

\begin{abstract}
Transmit preprocessing employed at the basestation (BS) has been proposed for simplifying the design of the mobile receiver. Provided that the channel impulse response (CIR) of all the BS to mobile station (MS) links is known in advance-even before the signal's transmission-it is plausible that the different users' signals may be differentiated with the aid of their unique, user-specific downlink CIRs. Naturally, this non-causal CIR knowledge is unavailable in practice. Hence a natural design option is to estimate the CIRs at the receiver after the BS's signal was received and convey it using side-information to the BS for its future use. Naturally, the resultant CIR has to be quantized before its transmission. In addition to this quantization error, it also becomes outdated and both imperfections result in an erosion of the achievable transmit preprocessing gain expressed in terms of either the attainable transmit power reduction or the number of users that may be supported. Another attractive design option is to avoid the CIR-signalling latency by invoking the previously received CIRs for predicting their future evolution using CIR-tap prediction. In this paper, Kalman filtering aided CIR prediction is combined with both minimum mean square error (MMSE) and zero forcing based preprocessing. Our simulation results show that the proposed scheme is capable of attaining $6.5 \mathrm{~dB}$ gain at a BER of $10^{-2}$, when using 6 antennas.
\end{abstract}

\section{INTRODUCTRON}

Practical communications systems have to support a multiplicity of users and hence diverse schemes have been proposed for supporting multiple users, including Time Division Multiple Access (TDMA), Code Division Multiple Access (CDMA) [1], Space Division Multiple Access (SDMA) [1] and so on. In CDMA systems, each user is assigned a unique user specific signature or spreading code, in order to differentiate them from the others, while in SDMA systems, the unique user specific spatial signature represented by the channel impulse response (CIR) acts like the unique spreading code of a CDMA system.

Recently, transmitter preprocessing techniques implemented at the BS have received wide attention [2]-[4], since they require a simple receiver at the mobile station (MS). The essential premise of using transmitter preprocessing techniques is the accurate knowledge of the CIR to be encountered at the transmitter, which requires sophisticated channel estimation. The accurate estimation of CIR at the MS's receiver and its signalling to the remote BS requires its high-precision signalling and inherently introduces delay, which may seriously degrade the attainable performance [4]. Alternatively, long-range channel prediction is an appropriate candidate for using already received past CIR estimates for the prediction of the CIR to be encountered during the next downlink (DL) transmission, which is capable of reducing the effects of the inherent delay [2], [3], when the BS has to await the MS's CIR estimates.

To elaborate a little further, explicit CIR signalling may be used in Frequency Division Duplex (FDD) systems, where the uplink and downlink operate at different carrier frequencies. By contrast, in Time Division Duplex (TDD) systems, the uplink and downlink signals are transmitted at the same carrier frequencies. Hence these signals experience similar CIRs as well as frequency domain channel transfer functions, unless their bandwidth is wider than the coherence

The financial support of the European Union under the auspices of the Newcom and Phoenix projects, as well as that of the EPSRC UK is gratefully acknowledged. bandwidth of the channel. The employment of a TDD mode is assumed in this paper.

In the context of MIMO channel prediction algorithms, the vector Kalman filtering assisted MIMO channel predictor has been successfully employed for tracking and predicting the MIMO channel [5]. Hence this technique is adopted in this paper, in order to facilitate BS transmitter preprocessing in the context of downlink TDD transmissons.

\section{SDMA DOWNLINK TRANSMISSION MODEL}

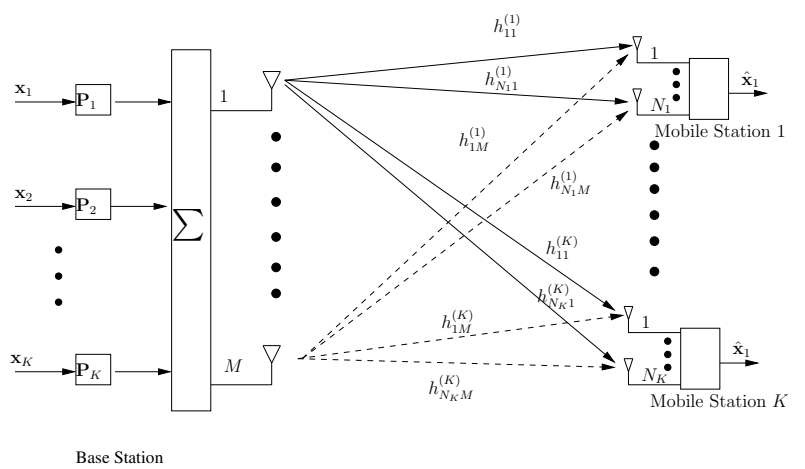

Fig. 1. Schematic of the SDMA downlink transmitter

Consider a system having a single BS and supporting $K$ MSs, as shown in Fig.1. The BS has $M$ transmitter antennas and the $k$ th MS has $N_{k} \geq 1$ receiver antennas. Furthermore, the channel between any pair of transmitter and receiver antennas is assumed to be flat-fading. The $N_{k}$-dimensional symbol vector $\mathbf{x}_{(d l, k)}$ is transmitted from the BS to the $k$ th MS, which can be expressed as

$$
\mathbf{x}_{(d l, k)}=\left[x_{(d l, k 1)}, x_{(d l, k 2)}, \cdots, x_{\left(d l, k N_{k}\right)}\right]^{T} .
$$

Before $\mathbf{x}_{(d l, k)}$ is transmitted, it is multiplied by the $\left(M \times N_{k}\right)$ dimensional transmit preprocessing matrix $\mathbf{P}_{k}$. Hence the preprocessed data vector $\mathbf{d}_{(d l, k)}$ is destined for the $k$ th user, which is given by

$$
\mathbf{d}_{(d l, k)}=\mathbf{P}_{k} \mathbf{x}_{(d l, k)} .
$$

Hence the $M$-dimensional composite preprocessed data vector $\mathbf{d}_{d l}$ of the $K$ users is given by

$$
\mathbf{d}_{d l}=\sum_{k=1}^{K} \mathbf{d}_{(d l, k)}=\mathbf{P} \mathbf{x}_{d l}
$$

where $\mathbf{P}$ is a $(M \times \mathcal{U})$-dimensional matrix and $\mathcal{U}=\sum_{k=1}^{K} N_{k}$, which is given by

$$
\mathbf{P}=\left[\mathbf{P}_{1}, \mathbf{P}_{2}, \cdots, \mathbf{P}_{K}\right],
$$

while $\mathbf{x}_{d l}$ is a $\mathcal{U}$-dimensional transmitted symbol vector, which has the structure of

$$
\mathbf{x}_{d l}=\left[\mathbf{x}_{(d l, 1)}^{T}, \mathbf{x}_{(d l, 2)}^{T}, \cdots, \mathbf{x}_{(d l, K)}^{T}\right]^{T} .
$$


The received $N_{k}$-dimensional vector $\mathbf{r}_{k}$ of the $k$ th user, is given by

$$
\begin{aligned}
\mathbf{r}_{k} & =\mathbf{H}_{k} \mathbf{d}_{d l}+\mathbf{n}_{(d l, k)} \\
& =\mathbf{H}_{k} \mathbf{P}_{k} \mathbf{x}_{(d l, k)}+\mathbf{H}_{k} \sum_{i=1, i \neq k}^{K} \mathbf{P}_{i} \mathbf{x}_{(d l, i)}+\mathbf{n}_{(d l, k)},
\end{aligned}
$$

where $\mathbf{n}_{(d l, k)}$ is an $N_{k}$-dimensional AWGN vector having zero mean and the autocorrelation matrix of $E\left[\mathbf{n}_{(d l, k)} \mathbf{n}_{(d l, k)}^{H}\right]=\sigma_{(d l, k)}^{2} \mathbf{I}_{N_{k}}$, while $\mathbf{H}_{k}$ is the $\left(N_{k} \times M\right)$-dimensional matrix of flat-fading CIR taps, whose $i$ th row, $j$ th column elements $h_{i j}^{(k)}$ represents the CIR coefficients between the $j$ th BS antenna and the $i$ th receiver antenna of the $k$ th MS. As we can see from (6), Multiple User Interference (MUI) is imposed on the $k$ th MS by all the other DL users.

The $\mathcal{U}$-dimensional received symbol vector $\mathbf{r}$ of the $K$ DL users can be expressed as

$$
\mathbf{r}=\left[\mathbf{r}_{1}^{T}, \cdots, \mathbf{r}_{K}^{T}\right]^{T}=\mathbf{H} \mathbf{P} \mathbf{x}_{d l}+\mathbf{n}_{d l},
$$

where $\mathbf{H}$ is a $(\mathcal{U} \times M)$-dimensional matrix, which is given by

$$
\mathbf{H}=\left[\mathbf{H}_{1}^{T}, \mathbf{H}_{2}^{T}, \cdots, \mathbf{H}_{K}^{T}\right]^{T},
$$

while $\mathbf{n}_{d l}$ is a $\mathcal{U}$-dimensional AWGN vector, which is expressed as

$$
\mathbf{n}_{d l}=\left[\mathbf{n}_{(d l, 1)}^{T}, \mathbf{n}_{(d l, 2)}^{T}, \cdots, \mathbf{n}_{(d l, K)}^{T}\right]^{T} .
$$

The AWGN has a zero mean and an autocorrelation matrix of

$$
E\left[\mathbf{n}_{d l} \mathbf{n}_{d l}^{H}\right]=\sigma_{d l}^{2} \mathbf{I}
$$

\section{MMSE CRITERION FOR DL PREPROCESSING}

Numerous criteria have been proposed for designing the DL preprocessing matrix $\mathbf{P}$ [2]-[4]. In this paper, the preprocessing matrix $\mathbf{P}$ is chosen based on the MMSE criterion, so that the MSE between the received signal vector $\mathbf{r}$ and the transmitted symbols $\mathbf{x}_{d l}$ expressed as $E\left[\left\|\mathbf{r}-\mathbf{x}_{d l}\right\|^{2}\right]$ is minimized.

\section{A. Exploiting the knowledge of the AWGN variance}

When the variance of the AWGN to be experienced at the MS's receiver can be correctly estimated and fed back to the BS via the uplink, the MSE between the received signal vector $\mathbf{r}$ and the transmitted symbols $\mathbf{x}_{d l}$ is expressed as [2]

$$
E\left[\left\|\mathbf{r}-\mathbf{x}_{d l}\right\|^{2}\right]=\operatorname{Trace}\left(\mathbf{R}_{\triangle}\right),
$$

where Trace $(\cdot)$ in (11) denotes the trace of the argument and $\mathbf{R}_{\triangle}$ is given by

$$
\mathbf{R}_{\triangle}=\mathbf{H} \mathbf{P} \mathbf{P}^{H} \mathbf{H}^{H}-\mathbf{H P}+\alpha \mathbf{P} \mathbf{P}^{H}-\mathbf{P}^{H} \mathbf{H}^{H}+\mathbf{I}_{\mathcal{U}},(12)
$$

where $\alpha$ is formulated as:

$$
\alpha=\frac{E\left[\mathbf{n}^{H} \mathbf{n}\right]}{E\left[\left|\mathbf{x}_{d l}\right|^{2}\right]}=\frac{\sigma_{d l}^{2}}{E_{s}}
$$

where $E_{s}$ denotes the power of each transmitted DL symbol and $\sigma_{d l}^{2}$ represents the AWGN variance at a MS's receiver, which is assumed to be the same for all MSs.

The problem of minimizing $E\left[\left\|\mathbf{r}-\mathbf{x}_{d l}\right\|^{2}\right]$ is now turned into minimizing the trace of $\mathbf{R}_{\triangle}$, which can be achieved by differentiating $\operatorname{Trace}\left(\mathbf{R}_{\triangle}\right)$ with respect to $\mathbf{P}^{*}$, yielding [6]

$$
\frac{\partial \operatorname{Trace}\left(\mathbf{R}_{\triangle}\right)}{\partial \mathbf{P}^{*}}=\mathbf{H}^{H} \mathbf{H} \mathbf{P}+\alpha \mathbf{P}-\mathbf{H}^{H} .
$$

Setting (14) to zero, we arrive at

$$
\begin{aligned}
\mathbf{P} & =\left(\mathbf{H}^{H} \mathbf{H}+\alpha \mathbf{I}_{M}\right)^{-1} \mathbf{H}^{H} \\
& =\mathbf{H}^{H}\left(\mathbf{H} \mathbf{H}^{H}+\alpha \mathbf{I}_{\mathcal{U}}\right)^{-1},
\end{aligned}
$$

where we have used the Matrix Inversion Lemma [7] in the second line of (15).

\section{B. Dispensing with the knowledge of the AWGN variance}

When the variance of the background AWGN to be experienced at the MS's receiver is unknown to the transmitter, the MSE between the received signal vector $\mathbf{r}$ and the transmitted symbols $\mathbf{x}_{d l}$ can be written as

$$
E\left[\left\|\mathbf{r}-\mathbf{x}_{d l}\right\|^{2}\right]=\operatorname{Trace}\left(\mathbf{R}_{\triangle}\right),
$$

where $\mathbf{R}_{\triangle}$ is a $(\mathcal{U} \times \mathcal{U})$-dimensional matrix, which can be expressed as

$$
\mathbf{R}_{\triangle}=\mathbf{H} \mathbf{P} \mathbf{P}^{H} \mathbf{H}^{H}-\mathbf{H P}+\sigma_{d l}^{2} \mathbf{I}_{\mathcal{U}}-\mathbf{P}^{H} \mathbf{H}^{H}+\mathbf{I}_{\mathcal{U}} .
$$

Comparison with (12) shows that there is no linkage between the AWGN variance $\sigma_{d l}^{2}$ and the preprocessing matrix $\mathbf{P}$ in (17). Similarly to (12), the specific solution which minimizes the trace of $\mathbf{R}_{\triangle}$ also minimizes the $E\left[\left\|\mathbf{r}-\mathbf{x}_{d l}\right\|^{2}\right]$. Hence, by differentiating $\operatorname{Trace}\left(\mathbf{R}_{\triangle}\right)$ with respect to $\mathbf{P}^{*}$, we have [6]

$$
\frac{\partial \operatorname{Trace}\left(\mathbf{R}_{\triangle}\right)}{\partial \mathbf{P}^{*}}=\mathbf{H}^{H} \mathbf{H} \mathbf{P}-\mathbf{H}^{H} .
$$

Setting (18) to zero, as in (14), yields

$$
\mathbf{H}^{H} \mathbf{H P}=\mathbf{H}^{H} \text {. }
$$

After further mathematical manipulations, we arrive at

$$
\mathbf{P}=\mathbf{H}^{+},
$$

where $\mathbf{H}^{+}$is the $(M \times \mathcal{U})$-dimensional pseudo inverse of the matrix $\mathbf{H}$, which is given by

$$
\mathbf{H}^{+}=\mathbf{H}^{H}\left(\mathbf{H H}^{H}\right)^{-1} .
$$

Upon substituting (21) into (7) we have

$$
\mathbf{r}=\mathbf{x}_{d l}+\mathbf{n}_{d l} .
$$

Observe from (22) that interestingly, we arrive at the zero-forcing preprocessing formulation despite using the MMSE criterion, when the variance of the noise is unavailable at the transmitter. Therefore, in the rest of this paper, we refer to preprocessing dispensing with the knowledge of the background AWGN variance as zero-forcing preprocessing. By contrast, preprocessing exploiting the knowledge of the receiver's background AWGN variance is termed as MMSE preprocessing.

\section{Power control}

It is a natural constraint that the transmitted power of all users should remain unchanged after preprocessing. In this case, the employment of power control has to be considered in the context of transmitter preprocessing and in fact the normalized preprocessing matrix $\mathbf{P}_{o}$ has to be used instead of $\mathbf{P}$ for the sake of satisfying the constraint of

$$
E\left[\left\|\mathbf{P}_{o} \mathbf{x}_{d l}\right\|_{2}^{2}\right]=E\left[\left\|\mathbf{x}_{d l}\right\|_{2}^{2}\right]=\mathcal{U} .
$$

A natural ambition is to allocate the total BS transmitter power to all the users employing the same normalized coefficient $\beta$ for all the users, yielding

$$
\mathbf{P}_{o}=\beta \mathbf{P},
$$

where $\beta$ is a real-valued variable. Upon substituting (24) into (23), we have

$$
\begin{aligned}
E\left[\left\|\mathbf{P}_{o} \mathbf{x}_{d l}\right\|_{2}^{2}\right] & =\operatorname{trace}\left(E\left[(\beta \mathbf{P}) \mathbf{x}_{d l} \mathbf{x}_{d l}^{H}(\beta \mathbf{P})^{H}\right)\right. \\
& =\beta^{2} \operatorname{trace}\left(\mathbf{P} \mathbf{P}^{H}\right) \\
& =\mathcal{U}
\end{aligned}
$$


Hence upon using (25) we have

$$
\beta=\sqrt{\frac{\mathcal{U}}{\operatorname{trace}\left(\mathbf{P P}^{H}\right)}} .
$$

\section{MIMO-AIDED SDMA UPLINK TRANSMISSION}

Let us now consider uplink (UL) transmissions, where $\mathbf{x}_{(u l, k)}=$ $\left[x_{(u l, k 1)}, x_{(u l, k 2)}, \cdots, x_{\left(u l, k N_{k}\right)}\right]^{T}$ is the $N_{k}$-dimensional UL transmitted symbol vector of the $k$ th user, as seen in Figure 2 Since TDD transmissions are considered in this paper, we assume that the UL and DL CIRs are indentical. Hence the $M$-dimensional received UL

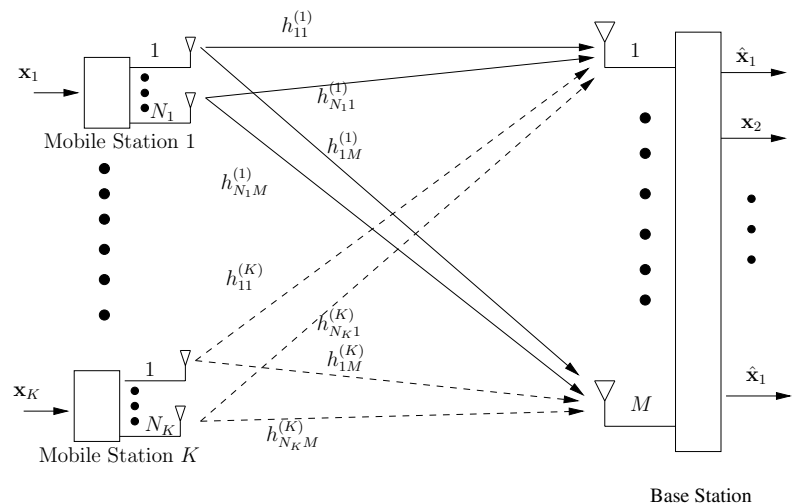

Fig. 2. Schematic of the SDMA uplink transmitter

signal $\mathbf{y}$ at $\mathrm{BS}$ is expressed as

$$
\mathbf{y}=\mathbf{H}^{T} \mathbf{x}_{u l}+\mathbf{n}_{u l},
$$

where $\mathbf{x}_{u l}=\left[\mathbf{x}_{(u l, 1)}^{T}, \mathbf{x}_{(u l, 2)}^{T}, \cdots, \mathbf{x}_{(u l, K)}^{T}\right]^{T}$ is the $\mathcal{U}$-dimensional composite transmitted symbol vector of the $K$ users, while $\mathbf{n}_{u l}$ is an $M$-dimensional AWGN vector having zero mean and an autocorrelation matrix of $E\left[\mathbf{n}_{u l} \mathbf{n}_{u l}^{H}\right]=\sigma_{u l}^{2} \mathbf{I}_{M}$.

\section{Channel Prediction Based SDMA DL Transmitter PREPROCESSING}

In order to perform BS transmit preprocessing for the SDMA downlink, the CIR matrix hosting DL CIRs must be available at the BS station. As a benefit of the TDD mode, the downlink CIRs can be estimated or predicted based on the CIRs of the uplink transmission 1 . However, the inherent delay of the estimated CIRs may seriously degrade the attainable performance [4]. Hence, the employment of prediction is preferred [2], [3] for the SDMA DL. In this paper, Kalman filtering assisted MIMO channel prediction is invoked [5]. To this end, (27) is rewritten for the time instant $n$ as

$$
\mathbf{y}(n)=\left[\mathbf{h}_{1}(n), \cdots, \mathbf{h}_{\mathcal{U}}(n)\right] \mathbf{x}_{u l}(n)+\mathbf{n}_{u l}(n),
$$

where the $M$-dimensional vector $\mathbf{h}_{j}(n), 1 \leq j \leq \mathcal{U}$ is the $j$ th column of $\mathbf{H}^{T}$, which represents the CIR vector between the $j$ th MS transmitter antenna and all the BS receiver antennas. Then, we construct a $(M \mathcal{U})$-length channel vector

$$
\mathbf{h}(n)=\left[\mathbf{h}_{1}^{T}(n), \mathbf{h}_{2}^{T}(n), \cdots, \mathbf{h}_{\mathcal{U}}^{T}(n)\right]^{T},
$$

Furthermore, the $i$ th, $1 \leq i \leq M$ element $h_{j i}(n)$ in $\mathbf{h}_{j}(n)$, which represents the CIR tap between the $j$ th MS transmitter antenna and

\footnotetext{
${ }^{1}$ As it was shown in Figure 15.3, p559 of [1] estimation, is carried out on the basis of previous CIR tap values for the current instant, while prediction determines their future values.
}

the $i$ th $\mathrm{BS}$ receiver antenna, can be described by an AutoRegressive (AR) model as [8]

$$
h_{j i}(n)=\sum_{q=1}^{p} a_{(j i, q)} h_{j i}(n-q)+w_{j i}(n),
$$

where $p$ is the order of the channel's AR model, $\left\{a_{(j i, q)}\right\}$ represents the AR model coefficients and $w_{j i}(n)$ is an AWGN process having a zero mean and an autocorrelation coefficient of $E\left[w_{j i}(n) w_{j i}^{*}(n)\right]=$ $\sigma_{w_{j i}(n)}^{2}$, where $\sigma_{w_{j i}(n)}^{2}$ is the variance of the modelling error of the AR model, which can be obtained from [8]. According to (30), the CIR vector between the $j$ th MS transmitter antenna and all the BS receiver antennas $\mathbf{h}_{j}(n)$ can be expressed as

$$
\mathbf{h}_{j}(n)=\sum_{q=1}^{p} \mathbf{A}_{(j, q)} \mathbf{h}_{j}(n-q)+\mathbf{w}_{j}(n),
$$

where $\left\{\mathbf{A}_{j, q}\right\}$ represents the $(M \times M)$-dimensional diagonal $\mathrm{AR}$ model coefficient matrices in the $j$ th column of the CIR tap matrix $\mathbf{H}^{T}$, which are given by

$$
\mathbf{A}_{j, q}=\left[\begin{array}{cccc}
a_{j 1, q} & 0 & \cdots & 0 \\
0 & a_{j 2, q} & \cdots & 0 \\
\vdots & \vdots & \ddots & \vdots \\
0 & 0 & \cdots & a_{j M, q}
\end{array}\right],
$$

while $\mathbf{w}_{j}(n)$ is an $M$-dimensional AWGN vector, which is given by

$$
\mathbf{w}_{j}(n)=\left[w_{j 1}(n), \cdots, w_{j M}(n)\right]^{T} .
$$

Upon substituting (31) into (29), the whole channel CIR vector $\mathbf{h}(n)$ can be expressed with the aid of AR model as

$$
\mathbf{h}(n)=\sum_{q=1}^{p} \mathbf{A}_{q} \mathbf{h}(n-q)+\mathbf{w}(n),
$$

where $\mathbf{A}_{q}$ is an $(M \mathcal{U} \times M \mathcal{U})$-dimensional diagonal matrix, which is expressed as

$$
\mathbf{A}_{q}=\left[\begin{array}{cccc}
\mathbf{A}_{1, q} & \mathbf{0} & \cdots & \mathbf{0} \\
\mathbf{0} & \mathbf{A}_{2, q} & \cdots & \mathbf{0} \\
\vdots & \vdots & \ddots & \vdots \\
\mathbf{0} & \mathbf{0} & \cdots & \mathbf{A}_{\mathcal{U}, q}
\end{array}\right],
$$

while $\mathbf{w}(n)$ is a $(M \times \mathcal{U})$-dimensional AWGN vector, which is given by

$$
\mathbf{w}(n)=\left[\mathbf{w}_{1}(n)^{T}, \cdots, \mathbf{w}_{\mathcal{U}}(n)^{T}\right]^{T} .
$$

If the $p$ CIR vectors $\mathbf{h}(n-q)(0 \leq q \leq(p-1))$ corresponding to $p$ consecutive time instants are combined into a new vector $\tilde{\mathbf{h}}(n)=$ $\left[\mathbf{h}(n)^{T}, \mathbf{h}(n-1)^{T}, \cdots, \mathbf{h}(n-p+1)^{T}\right]^{T}$, based on (34) we arrive at

$$
\tilde{\mathbf{h}}(n)=\tilde{\mathbf{F}}(n) \tilde{\mathbf{h}}(n-1)+\tilde{\mathbf{w}}(n),
$$

where $\tilde{\mathbf{F}}(n)$ is a $[(M \times \mathcal{U} \times p) \times(M \times \mathcal{U} \times p)]$-dimensional transition matrix [7], describing the state transition from time instant $(n-1)$ to $n$, which is given by

$$
\tilde{\mathbf{F}}_{n}=\left[\begin{array}{cccc}
\mathbf{A}_{1} & \mathbf{A}_{2} & \ldots & \mathbf{A}_{p} \\
\mathbf{I}_{(M \times \mathcal{U})} & \mathbf{0} & \cdots & \mathbf{0} \\
\vdots & \ddots & \ddots & \vdots \\
\mathbf{0} & \cdots & \mathbf{I}_{(M \times \mathcal{U})} & \mathbf{0}
\end{array}\right],
$$

while $\tilde{\mathbf{w}}(n)$ is a $((M \times \mathcal{U} \times p)$-dimensional noise vector, which is formulated as

$$
\tilde{\mathbf{w}}(n)=\left[\mathbf{w}(n)^{T}, \mathbf{0}^{T}, \cdots, \mathbf{0}^{T}\right]^{T} .
$$




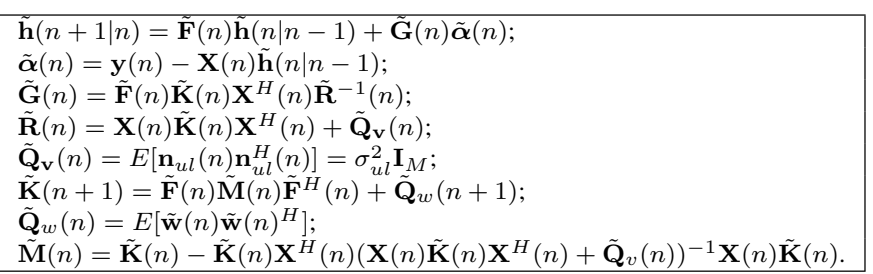

TABLE I

Vector Kalman Filtering Assisted Mimo Channel Prediction

Now the received UL signal vector of (28) can be rewritten as [5]

$$
\mathbf{y}(n)=\mathbf{X}(n) \tilde{\mathbf{h}}(n)+\mathbf{n}_{u l}(n),
$$

where $\mathbf{X}$ is a $(M \times(M \times \mathcal{U} \times p))$-dimensional matrix, which is given by

$$
\mathbf{x}=\tilde{\mathbf{x}} \bigotimes \mathbf{I}_{M},
$$

with $\otimes$ representing the Kronecker product. Furthermore, in (41) $\tilde{\mathbf{x}}$ is a $(\mathcal{U} \times p)$-dimensional row vector, given by

$$
\tilde{\mathbf{x}}(n)=\left[\mathbf{x}_{u l}^{T}, \mathbf{0}\right] \text {. }
$$

Given the process equation and measurement equation of (37) and (40) [7], respectively, vector Kalman filtering assisted MIMO channel prediction of $\tilde{\mathbf{h}}(n+1 \mid n)$ based on all the observations up to the time instant $n$, can be performed using the vector-based Kalman filtering assisted MIMO CIR-tap prediction procedure described in Table I [7], [9].

\section{Performance Results}

In this section, we provide simulation results for characterizing the performance of channel prediction aided downlink preprocessing. More specifically, a generalized TDD mode is assumed, where the time-span between two transmitted uplink symbols used for CIR tap prediction is assumed to be $L$ and the range of prediction $\mathcal{L}$, where the predicted CIR taps are used is shown in Figure 3. Furthermore, $T_{s}$ is the symbol duration, while the normalized maximum Doppler frequency is given by $f_{d m} T_{s} L$. Additionally, BPSK modulation is employed for both uplink and downlink transmissions. Finally, errorfreely detected uplink symbols are assumed instead of invoking any specific uplink detector, implying that our results represent an idealistic upper bound.

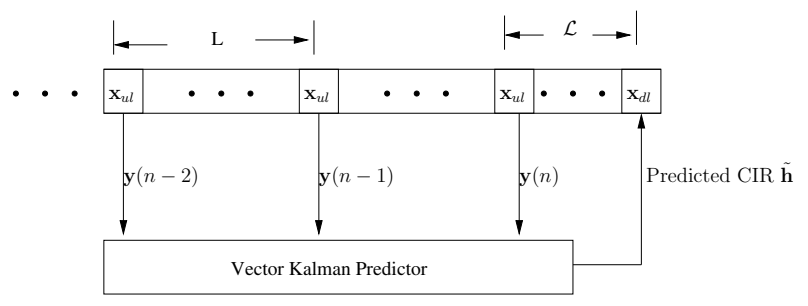

Fig. 3. Schematic of the MIMO channel prediction scheme porposed for TDD systems.

\section{A. Effect of the number of transmitter antennas at the BS}

In Figure 4, the attainable BER performance versus average SNR is plotted for the first receive antenna of the first user, when the number of antennas used for MMSE BS transmit preprocessing is assumed to be $M=4,6,8$, and 10, respectively. Furthermore,

\begin{tabular}{|l|r|}
\hline Number of users $K$ & 2 \\
\hline Number of antennas per user $N_{k}$ & 2 \\
\hline Order of Kalman filtering assisted predictor $p$ & 2 \\
\hline Range of prediction $\mathcal{L}$ & $\mathrm{L} / 2$ \\
\hline
\end{tabular}

TABLE II

PARAMETERS OF THE CHANNEL PREDICTION BASED SDMA DOWNLINK PREPROCESSING IN TDD MODE

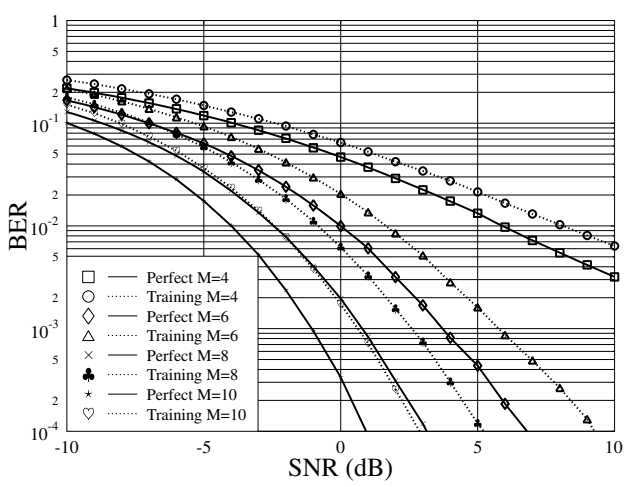

Fig. 4. BER versus average SNR per symbol performance for downlink transmission for the first receive antenna of the first user, when the number of transmitter antennas at the BS is assumed to be 4, 6, 8, and 10, respectively for MMSE BS transmit preprocessing. Furthermore, the maximum normalized Doppler frequency is $f_{d m} T_{s} L=0.001$ and the remaining parameters are the same as in Table II.

the maximum normalized Doppler frequency is $f_{d m} T_{s} L=0.001$ and the remaining parameters are the same as in Table II. As we can see from Figure 4, once the number of transmitter antennas at the BS becomes more than the sum of the number of each MS's receiver antennas, the achievable BER performance is significantly improved for both the perfect and predicted CIR-tap scenarios as a benefit of tranmitter diversity. Furthermore, observe in Figure 4 that the performance difference between $M=4$ and $6, M=6$ and 8 , as well as $M=8$ and 10 recorded for both the perfect and predicted CIR-tap scenarios, becomes narrower. This is because the achieveable extra additional transmit diversity gain becomes lower upon every further increase of the number of antennas $M$ at the BS. Moreover, the performance discrepancy seen in Figure 4 between the perfect and predicted CIR-tap scenarios becomes lower upon increasing the number of antennas at the BS since the performance loss due to prediction can be effectively compensated by increasing the transmit diversity gain and hence approaching a near AWGN performance. Additionally, the performance discrepancy between the scenarios using predicted CIR taps in conjunction with $M=10$ and perfect CIR taps with $M=8$ is narrower than that between the predicted CIR scenario using $M=8$ and the perfect CIR scenario employing $M=6$. Similarly, observe in Figure 4 that the latter scenario has a lower performace discrepancy than that between the predicted CIR case employing $M=6$ and the perfect CIR scenario using $M=4$. Especially, the BER performance of the predicted CIR scenario using $M=10$ was shown to be better in Figure 4 than that using perfect CIR-tap knowledge in conjunction with $M=8$ in the lower range of SNRs. The reason for this is because the extra transmit gain attained compensates for the performance loss imposed by the CIR-tap prediction error. The same trend can be observed in 
the context of zero-forcing aided BS transmit preprocessing.

\section{B. Performance with or without the knowledge of AWGN variance}

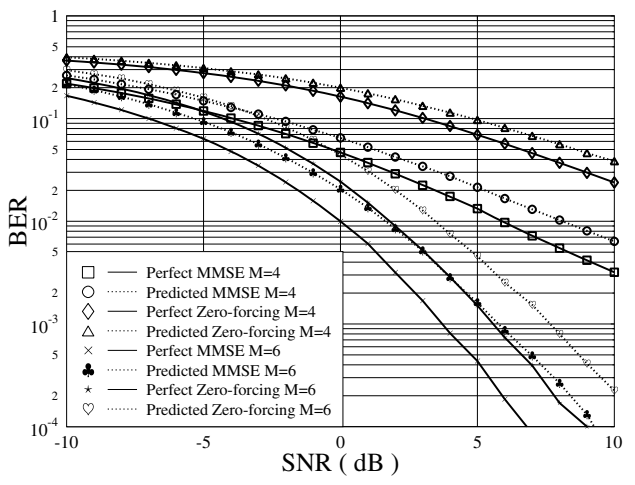

Fig. 5. BER versus average SNR per symbol performance for downlink transmission for the first receive antenna of the first user when the number of transmitter antennas at BS is assumed to be 4 and 6, respectively, for both the MMSE and zero-forcing BS transmit preprocessing. Furthermore, the maximum normalized Doppler frequency is $f_{d m} T_{s} L=0.001$. The remaining parameters are the same as in Table II.

In Figure 5 the BER versus average SNR per symbol performance was recorded for downlink transmission in the context of the first receive antenna of the first user when the number of antennas at the BS is assumed to be $M=4$ and 6, respectively, for both MMSE and zero-forcing BS transmit preprocessing. Furthermore, the maximum normalized Doppler frequency is $f_{d m} T_{s} L=0.001$. We can see from Figure 5 that as expected, the BER performance of both MMSE and zero-forcing BS transmit preprocessing is improved upon increasing the SNR, regardless whether perfect or prediced CIR taps are used. Apart from the obvious effect of noise reduction at the detector, this BER performance improvement is also due to the associated more accurate prediction, when using predicted CIR taps. Moreover, observe in Figure 5 for a given number of antennas at the BS the achievable BER performance of MMSE preprocessing is better than that of zero-forcing preprocessing for both the perfect and predicted CIR scenarios. This is because the effects of both the MUI and AWGN are jointly taken into account by the MMSE preprocessing. Furthmore, the performance discrepancy between MMSE and zeroforcing preprocessing recorded in Figure 5 for both the perfect and predicted CIR taps scenarios becomes narrower upon increasing the number of transmitter antennas from $M=4$ to 6 . This is because zero-forcing preprocessing benefits more from the associated increased transmit diversity than MMSE preprocessing, since the transmitter diversity significantly mitigates not only the effect of the fading, but also those of the AWGN, as a benefit of noise averaging. Additionally, the discrepancy between the MMSE and zero-forcing based preprocessing techniques becomes narrower upon increasing the SNR for both the perfect and prediced CIR taps scenarios when considering the same number of antennas at BS. This is because MMSE preprocessing loses its benefits of mitigating the effects of the AWGN upon increasing the SNR. Moreover, as seen in Figure 5 the MMSE preprocessing using $M=4$ transmitter antennas outperforms the zero-forcing scheme for both the perfect and predicted CIR-tap scenarios, regardless of the SNR. Moreover, the MMSE preprocessor using $M=4$ transmitter antennas outperforms the zero-forcing scheme, even when $M=6$ tranmitter antennas are used in both the perfect and predicted CIR scenarios in the lower SNR range of Figure 5. This is because the MMSE preprocessing has the ability of mitigating the effects of the AWGN. Furthermore, for $M=6$ transmitter antennas the MMSE preprocessor using predicted CIR taps becomes capable of outperforming the zero-forcing preprocessor benefitting from perfect CIR tap in the lower range of SNR of Figure 5. The reason for this is because the detrimental effects of CIR-tap prediction error are lower for MMSE preprocessing than the effects of the AWGN imposed on zero-forcing preprocessing using perfect CIR taps, especially for lower SNR range of Figure 5.

\section{CONCLUSION}

Transmitter preprocessing has been investigated as an key technique of simplifying the MS's receiver. A crucial requirement for its success is the accurate and prompt knowledge of the CIR taps at the BS. The quantized and outdated CIR tap knowledge results in a performance degration. Hence, CIR-tap prediction becomes an essential technique in this situation. Furthermore, the CIR tap values extracted from the uplink transmissions can be used for predicting the downlink CIR taps in TDD systems. In this paper, a TDD based SDMA system using a vector Kalman filtering assisted predictor was used at the BS for predicting the downlink CIR taps in order to invoke transmitter preprocessing. The MMSE criterion was adopted for designing the BS's transmit preprocessing matrix and two different forms of preprocessing, namely MMSE and zero-forcing were used corresponding to known or unknon background AWGN variance, respectively. Our simulation results in Figures 4 to 5 demonstrated that with the aid of the Kalman filtering assisted channel predictor, BS transmitter preprocessing is capable of achieving an attractive performance. Furthermore, MMSE preprocessing was shown to outperform zero-forcing preprocessing benefitting from its ability of mitigating the AWGN, as seen in Figure 5.

\section{REFERENCES}

[1] L. Hanzo, M. Münster, B. Choi, and T. Keller, OFDM and MC-CDMA for Broadband Multi-User Communications, WLANs and Broadcasting. Wiley, 2003.

[2] R. L. Choi and R. D. Murch, "New transmit schemes and simplified receivers for MIMO wireless communication systems," IEEE Transactions on Wireless Communications, vol. 2, pp. 1217- 1230, November 2003.

[3] R. Choi and R. Murch, "MIMO transmit optimization for wireless communication systems," in The First IEEE International Workshop on Electronic Design, Test and Applications, (Christchurch, New Zealand), pp. 33 - 37, 29 - 31 January 2002.

[4] L. Choi and R. D. Murch, "Transmit-preprocessing techniques with simplified receivers for the downlink of MISO TDD-CDMA systems," IEEE Transactions on Wireless Communications, vol. 35, pp. 285- 295, March 2004.

[5] C. Komninakis, C. Fragouli, A. H. Sayed, and R. D. Wesel, "Multiinput multi-output fading channel tracking and equalization using Kalman estimation," IEEE Transactions on Signal Processing, vol. 50, pp. 1065 - 1076, May 2002.

[6] D. H. Brandwood, "A complex gradient operator and its apphcation in adaptive array theory," IEE Proceedings, Part F - Communications, Radar and Signal Processing, vol. 130, pp. 11 - 16, February 1983.

[7] S. Haykin, Adaptive Filter Theory. Prentice Hall, Inc, fourth ed., 2002.

[8] K. E. Baddour and N. C. Beaulieu, "Autoregressive modeling for fading channel simulation," IEEE Transactions on Wireless Communications, vol. 4, pp. 1650 - 1662, July 2005.

[9] R. G. Brown and P. Y. C. Hwang, Introduction to Random Signals and Applied Kalman Filtering: With Matlab Exercises And Solutions. Wiley, third ed., 1997. 